Integrierte Produktpolitik in Europa - Initiativen und Herausforderungen

\section{Auf halber Wegstrecke}

Die Umweltpolitik widmet sich verstärkt Produkten und Dienstleistungen. Neu ist dabei ein integrierender und systematisierender Ansatz, der in den letzten Jahren unter dem Stichwort „Integrierte Produktpolitik“" bekannt geworden ist. Inzwischen liegen in verschiedenen Ländern Europas Erfahrungen vor. Auch in Deutschland hat die Diskussion begonnen, aber die entscheidenden Ausgestaltungsfragen sind noch zu klären.

$\mathrm{D}$ Von Frieder Rubik letzten Jahre haben eine Vielzahl von Erfolgen im prozess- und anlagenbezogenen Umweltschutz erbracht. Diese Erfolge, der wirtschaftliche Strukturwandel und das Wachstum des privaten Endverbrauchs lenkten den Blick auf Umweltbelastungen durch Produkte und Dienstleistungen entlang ihres gesamten stofflichen Lebenswegs. Dieser geht von der Rohstoffgewinnung über Produktion, Handel und Nutzung bis hin zur Entsorgung. Diese umfassende Perspektive ist der Ausgangspunkt von Integrierter Produktpolitik (IPP)

Der Begriff IPP wurde in einem Gutachten im Auftrag der EU-Generaldirektion (DG) Umwelt eingeführt und definiert als: „Public policy which explicitly aims to modify and improve the environmental performance of product systems" (1). Mittlerweile nahmen eine Vielzahl unterschiedlicher Kreise dazu Stellung. Das Institut für ökologische Wirtschaftsforschung (IÖW) schlug jüngst in einem Gutachten für das deutsche Bundesumweltministerium folgende Definition vor: „IPP setzt an Produkten und Dienstleistungen und deren ökologischen Eigenschaften entlang des gesamten Lebensweges an; sie zielt auf die Verbesserungen ihrer ökologischen Eigenschaften ab und fördert hierzu Innovationen von Produkten und Dienstleistungen“ (2).

\section{IPP zwischen Markt und Staat?}

Vielfach wird einer solchen Zugangsweise aus Kreisen der Wirtschaft jedoch mit Misstrauen begegnet: Übernimmt nun der Staat die betrieblichen Produktentscheidungen? Was bleibt dann noch von der Marktwirtschaft übrig? Diese Befürchtungen sind sicherlich ernst zu nehmen, sie verkennen aber auch Möglichkeiten. Zweifelsohne haben viele Unternehmen die ökologische Fitness ihrer Produkte verbessert - und zwar nicht nur Anbieter von Nischenprodukten, son- dern auch Hersteller von Produkten, die die Massenmärkte bedienen. Auch mit Blick auf einschlägige Untersuchungen zum Zustand der Umwelt kann gleichwohl kaum davon gesprochen werden, dass nicht noch weitere und möglicherweise sehr große Verbesserungen notwendig sind, um eine nachhaltige Entwicklung voran zu bringen. Dabei sollte auch produktseitig dem Lebensweggedanken gefolgt werden. Alle Beteiligten stehen vor der Herausforderung, hierzu Beiträge zu leisten.

Staatlicherseits kann dies über klare, verlässliche, konsistente und kalkulierbare Rahmenbedingungen und Zielsetzungen sowie verschiedene Aktivitäten erfolgen. Damit kann der Markt mit seinen verschiedenen Akteuren unterstützt werden, eine ökologische Transformation zu verfolgen. Der zentrale Zugang hierzu ist die Unterstïtzung bzw. Mobilisierung von Innovationen, die Produkte wie auch Dienstleistungen ökologisch fit machen (vgl. dazu auch den Beitrag von René Kemp in diesem Heft). Derartige konzeptionelle Überlegungen werden seit Anfang der 90er Jahre in einer Reihe von europäischen Ländern sowie von der Europäischen Union (EU) angestellt.

\section{Die Rolle der EU}

Beispiele für Aktivitäten und Maßnahmen der Europäischen Union im Bereich des produktbezogenen Umweltschutzes sind das EU-Umweltzeichen oder Initiativen im Bereich der Forschung. Diese Maßnahmen waren jedoch in erster Linie singuläre „Ereignisse“. Im Zuge der Umsetzung des fünften Umweltaktionsprogramms der $\mathrm{EU}$, das für den Zeitraum 1992 bis 2000 gültig war, wurde erkannt, dass ein konzeptioneller und systematischer Zugang zu Produkten notwendig war. Hierzu wurde 1996 die Unternehmensberatung Ernst\&Young und die Universität von Sussex mit einem Gutachten zu einer Produktpolitik beauf- tragt. Der Bericht wurde 1998 vorgelegt und auf einem Workshop in Brüssel präsentiert. Er löste eine intensive Diskussion auf Workshops und innerhalb verschiedener gesellschaftlicher Anspruchsgruppen aus (3). Auf einem informellen EU-Umweltministerrat im Mai 1999 wurde IPP als ein Schwerpunkt behandelt und seitens der Umweltminister ein Aufgabenpaket für die Kommission geschnürt (4).

Mittlerweile werden innerhalb der DG Umwelt die Arbeiten vorangetrieben, ein politisches Diskussionspapier in Form eines sogenannten Grünbuchs zu IPP vorzulegen. Mit einer Veröffentlichung ist frühestens Ende November zu rechnen. Im Frühjahr 2001 soll das Werk auf einem Treffen mit interessierten Kreisen und einem EU-Umweltministerrat diskutiert werden. Ob das Grünbuch in ein Weißbuch, also eine EU-Konzeption für IPP, überführt wird, ist allerdings offen.

Außerdem möchte die DG Umwelt nächstes Jahr einige Pilotprojekte zu einer IPP auf freiwilliger Basis initiieren. Innerhalb des sechsten Umweltaktionsprogramms, das derzeit vorbereitet und bis zum Jahre 2010 gelten wird, soll IPP eine prominente Rolle einnehmen. Eine Fortentwicklung der IPP auf Ebene der EU ist auch durch die EU-Ratspräsidentschaft Schwedens im ersten Halbjahr 2001 zu erwarten.

\section{Europäische Vorreiter}

Dänemark, Großbritannien, die Niederlande (vgl. den Artikel von Henk Wijnen) sowie Schweden (vgl. den Artikel von Eva Ablner et al.) haben eigenständige politische Dokumente erarbeitet, die als Konzeptionen einer IPP verstanden werden können. Die folgende Tabelle gibt einen Überblick über den Stand der Aktivitäten in diesen vier Ländern. Daneben gibt es Bemühungen in Deutschland (siehe unten) sowie in Finnland, Frankreich, Italien und Österreich, die bisher jedoch noch nicht in Konzeptionen gemündet sind.

Vergleicht man die Lage in den vier Vorreiterstaaten, so ergibt sich folgendes Bild:

- Die wesentlichen Prinzipien und die Steuerungsobjekte scheinen überein zu stimmen. Allerdings fällt auf, dass Dienstleistungen in fast keiner Konzeption explizit erwähnt werden.

- Einigkeit besteht ebenfalls hinsichtlich der Zielorientierung, nämlich der Entlastung der Umwelt. Dieser allgemeine Konsens wird jedoch kaum näher konkretisiert.

- Die vier Staaten weisen beträchtliche Unterschiede hinsichtlich der konkreten Umsetzung und Implementation ihrer jeweiligen Konzeptio- 


\begin{tabular}{|c|c|c|c|c|}
\hline Merkmal & Dänemark (5) & Großbritannien (6) & Niederlande $(7)$ & Schweden (8) \\
\hline Steuerungsobjekte & Produkte & Endverbrauchernahe Produkte & Produkte & Produkte und Dienstleistungen \\
\hline \multirow[t]{7}{*}{ Ziele } & - Entwicklung und Vermarktung & Markttransformation durch & Verbesserung der ökologischen & - Verbesserung der ökologischen \\
\hline & von ökologischen Produkten & - Umweltentlastungen & Eigenschaften von Produkten & Eigenschaften von Produkten \\
\hline & - Verringerung von & - Innovationen & & und Dienstleistungen \\
\hline & Umweltbelastungen & & & - Stärkung der europäischen \\
\hline & - Konsolidierung der & & & Wettbewerbsfähigkeit \\
\hline & Wettbewerbsfähigkeit der & & & \\
\hline & dänischen Wirtschaft & & & \\
\hline \multirow[t]{5}{*}{ Prinzipien } & - Berücksichtigung der & - Marktkompatibilität & Ergebnis- statt & - Allgemeine umweltpolitische \\
\hline & Bedürfnisse der Anspruchsgruppen & - Ergebnis- statt & Instrumentenorientierung & Prinzipien der EU und Schweden \\
\hline & - Kompatibilität mit dänischen und & Methodenorientierung & & - Integration entlang des \\
\hline & internationalen Marktbedingungen & - Flexibilitüt und Selektivitüt & & Produktlebenswegs \\
\hline & & bei Instrumentenwahl & & - Einbezug der Akteure \\
\hline \multirow[t]{2}{*}{ Instrumente } & Umfassend, aber Konzentration & Umfassend, kein Ausschluss & Primär finanzielle, technische & Umfassend, kein \\
\hline & auf ausgewählte Maßnahmen & bestimmter Instrumente & Unterstützung der Unternehmen & Ausschluss bestimmter Instrumente \\
\hline \multirow[t]{2}{*}{ Orientierung } & Angebots- und Nachfrageseite & Angebots- und Nachfrageseite; & Angebotsseite & Angebots- und Nachfrageseite \\
\hline & & bei Maßnahmen primär letztere & & \\
\hline \multirow[t]{4}{*}{ Schwerpunkte } & Mehrere; keine eindeutigen Prioritäten & - Endverbrauchernahe Produkte & Förderung der Produktaktivitäten & - Informationstransmission \\
\hline & & - Produktinformationen & in Unternehmen & und -diffusion \\
\hline & & & & - Zielvorgaben an verschiedene \\
\hline & & & & Akteure \\
\hline Prioritäre & Product panels tür Textilien, & Nicht explizit & Nicht explizit & Keine \\
\hline Produktgruppen & Elektronik, Transport und Bauen & & & \\
\hline \multirow[t]{11}{*}{ Maßnahmen } & - Daten für Okobilanzen & - Verpflichtendes & - Förderung von Okodesign & - Umweltzeichen \\
\hline & - Unterstützung der Anwendung & Energieverbrauchskennzeichen & - Förderung von produktbezogenen & - Quantitative Produktinformationen \\
\hline & von Ökobilanzen & - Freiwillige Kennzeichen durch & Umweltmanagementsystemen & - Förderung des \\
\hline & - Umweltkennzeichnung & Verbände und Unternehmen & & Umweltmanagements \\
\hline & - Produkthandbücher & - Ökologisierung der Produktnormen, & & - Unterstützung des öffentlichen \\
\hline & - Öffentliches Beschaffungswesen & eigene ökologische Produktnormen & & Beschaffungswesens \\
\hline & - Ökostevern & - Umweltpreise & & - F\&E Programme und Förderung \\
\hline & - Finanzhilfen & - Umweltzeichen ISO Typ I & & - Normung \\
\hline & - Zielvorgabe (Liste unerwünschter & & & - Prüfung der Ausweitung der \\
\hline & Substanzen) & & & Produzentenverantwortung \\
\hline & & & & - Diverse EU-Aktivitäten \\
\hline
\end{tabular}

nen auf: Manche Staaten verfolgen eine Orientierung auf die Angebotsseite, während in anderen die Nachfrageseite im Vordergrund steht. Die nationalen Maßnahmen, Aktivitäten und Schwerpunktsetzungen unterscheiden sich dementsprechend beträchtlich.

- Während die Sicht auf die möglichen IPP-Instrumente im allgemeinen recht breit und auch jeweils ähnlich ist, werden in der Umsetzung länderspezifische Schwerpunkte gesetzt, die zu beträchtlichen Unterschieden führen.

Diese Trends unter den EU-Mitgliedsstaaten weisen der EU-Kommission eine beträchtliche Verantwortung zu. Ihre Aufgabe ist es die Pluralität der Ansätze zusammenzuführen.

\section{IPP in Deutschland}

Maßnahmen eines produktbezogenen Umweltschutzes reichen in Deutschland bis in die 70er Jahre zurück. Innerhalb vieler Branchen gibt es bereits zahlreiche Maßnahmen seitens der Umweltpolitik wie auch seitens der Wirtschaft (vgl. den Beitrag von Esther Hoffmann und Ulla Simshäuser). Insgesamt hat sich eine Vielzahl von Ansätzen, Instrumenten, Zugangsweisen und Traditionen herausgebildet, die jedoch noch auf keinem konzeptionellen Gerüst aufbauen können und denen eine Systematisierung fehlt. Die Arbeiten des Umweltbundesamtes (UBA) und des Bundesumweltministeriums (BMU) lassen entsprechende Orientierungslinien in Bezug auf eine IPP erkennen. Das UBA beschäftigte sich insbesondere mit Ökobilanzen und Stoffstrommanagement, während das BMU Vorarbeiten zu einer deutschen IPP realisierte.

Mit der aufkommenden europäischen Diskussion wurde ein zweiter Anlauf unternommen: Das Heidelberger Büro des IÖW erarbeitete 1999 und 2000 im Auftrag des BMU konzeptionelle und instrumentelle Vorschläge zu einer Konkretisierung einer IPP auf deutscher Ebene (9).

Ein für die deutsche IPP-Diskussion bemerkenswertes Ereignis war der Workshop „Perspektiven des produktbezogenen Umweltschutzes“, den das BMU und der Bundesverband der deutschen Industrie (BDI) am 1. Februar 2000 gemeinsam veranstaltet haben. Hierbei zeigte sich ein große Ge- 
politische ökologie

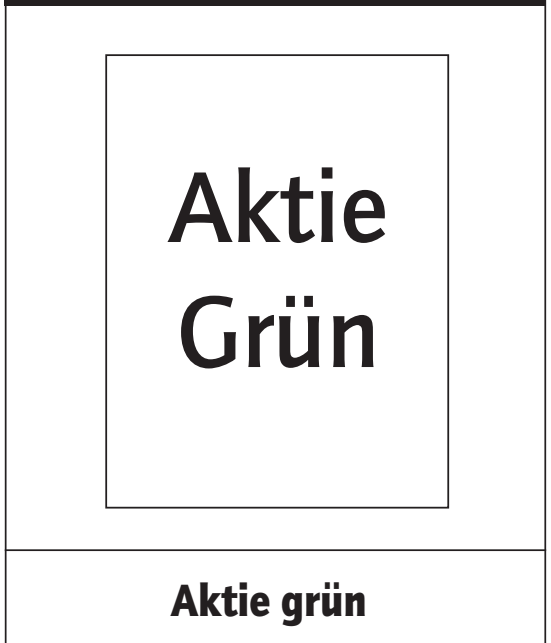

Die Tage, an denen man Investitionsentscheidungen unabhängig von ökologischen, ethischen und sozialen Konsequenzen treffen konnte, sind gezählt. Banken erkennen zunehmend, dass ihr Geschäftserfolg wesentlich von der Berücksichtigung ökologischer Kriterien abhängt. Auch der Aktienmarkt wird grüner: Neue Fonds sprießen aus dem Boden, die nach ethisch-ökologischen Kriterien augerichtet sind. Hinzukommt, dass nicht nur Kleinanleger sich mehr und mehr dafür interessieren, wohin ihr Geld fließt und was es bewirkt. Auch institutionelle Anleger und Vermögensverwalter lernen nach und nach, dass sich mit ethischen Werten Geld verdienen lässt.

Grund genug für die poltische ökologie dieses Thema erneut aufzugreifen. Nachdem 1997 mit "Wechsel ohne Deckung" (politische ökologie 53) der grundlegende Zusammenhang zwischen Geld und Ökologie im Zentrum stand, liegt der Focus nun auf der sich ausbreitenden ethisch-ökologischer Aktienkultur. Namhafte Autoren diskutieren und beleuchten das Thema ideenreich aus unterschiedlichen Blickwinkeln.

Mit Beiträgen von Jörg Weber, Prof. Henry Schäfer, Reinier de Man, Axel Wilhelm, Oliver Karius, Prof. Johannes Hoffmann, Robert Haßler, Babara Wieler, Reinhard Loske, Werner Nussbaum, Christian Armburster, Elmar Sing u.a.

\section{www.oekom.de}

Jetzt für 29,80 Mark direkt

zu bestellen bei:

pan adress

politische ökologie Leserservice

Semmelweisstr. 8

D-82152 Planegg

Fon $++49|(0) 89| 85709-145$

Fax $++49|(0) 89| 85709-131$ meinsamkeit in der Bewertung der Ausgangslage. So wurden mehrfach, z.B. von Hans-Olaf Henkel als Präsident des BDI wie auch vom Leiter der Grundsatzabteilung des BMU, Andreas Gallas, drei Schlüsselbegriffe für eine IPP genannt, nämlich Integration, Kooperation und Kommunikation. Henkel betonte, dass sowohl Politik wie auch Wirtschaft Aufgaben anzupacken hätten, einen Dialog führen wie auch den europäischen Rahmen einer IPP nachhaltig mitgestalten sollten (vgl. zur Sicht des BDI auch den Beitrag von Thomas Becker). Allgemein ist Deutschland aufgrund seiner Tradition und seiner Vielfalt an instrumentellen Zugängen zu den Vorreitern einer IPP innerhalb Europas zu zählen. Gleichwohl besteht weiterhin die Aufgabe, eigenständige nationale Überlegungen seitens der Politik anzustellen, um im europäischen Diskurs mit den anderen Mitgliedsländern der EU „,mithalten“ zu können. Überlegungen aus dem BMU hierzu findet man in dem Beitrag von Eckart Meyer-Rutz.

\section{- Bevorstehende Aufgaben}

Die zentrale Herausforderung auf europäischer und deutscher Ebene ist die konzeptionelle Präzisierung einer IPP. Hier sind Eckpunkte und Leitplanken notwendig, um dem Markt und den verschiedenen direkt bzw. indirekt darauf Einfluss nehmenden Akteuren ökologische Signale zu geben. Zentrale Botschaft muss die Orientierung einer IPP auf Innovationen und die Verfolgung entsprechender unterstïtzender Maßnahmen sein.

Gleichwohl ist die derzeitige IPP-Diskussion noch von einer gewissen Abstraktheit gekennzeichnet. Eine „Erdung“ und Übersetzungsarbeit in Form von Pilotprojekten scheint in diesem Kontext ratsam, um Erfahrungen zusammenzutragen und den Mehrwert einer IPP klarzumachen. Insofern ist es zu begrüßen, wenn entsprechende Aktivitäten auf deutscher wie auch auf europäischer Ebene für das nächste Jahr zu erwarten sind. Hierbei gilt es,

\section{Einen Überblick über alle bisher} erschienen Hefte des Informationsdienstes „Ökologisches Wirtschaften" finden Sie oư unseren

Internetseiten unter der Adresse: http://www.oekom.de Wir freuen uns auf hren Besuch!
Erfahrungen und Möglichkeiten einer IPP anhand ausgewählter Bedarfsfelder oder Produktgruppen sorgfältig zu analysieren und zu evaluieren und den kooperativen IPP-Ansatz empirisch auszutesten (vgl. den Beitrag von Hoffmann/Simshäu$s e r)$. Derartige Pilotprojekte sollten insbesondere die change agents - also die Innovationspromotoren - einbeziehen, die als Antreiber für ökologische Innovationen innerhalb der Branche fungieren könnten.

Diese Aufgabe richtet sich sowohl an die PraxisAkteure wie auch an die Wissenschaft. Letztere hat zwar vielfach auf den Charme der Produktorientierung hingewiesen, muss aber noch die Antwort auf die Frage liefern, wie dies in der Realität wirklich gelingen kann. Dann könnte sich auch der vermeintliche Antagonismus zwischen einem „Top-down“ und einem „Bottomup“-Ansatz der IPP auflösen.

\section{Anmerkungen}

(1) Ernst \& Young / SPRU: Integrated Product Policy. Report to the EU, London 1998, S. 33. Kurzfassung unter http://europa.eu.int/comm/environment/ipp/home.htm (2) Rubik, Frieder: Innovationen durch die Umweltpolitik - Integrierte Produktpolitik (IPP) in Deutschland. IÖW, Heidelberg/ Berlin 2000, S. 27.

(3) Vgl. zum Entstehungsprozess von IPP auch Rubik, Frieder/ Simshäuser, Ulla: Dynamischer Prozess. Entwicklung und Perspektiven integrierter Produktpolitik. In: Ökologisches Wirtschaften, Heft 5-6/1999, S. 8-9.

(4) Ergebnisse des Ministerrats und das Hintergrundpapier sind in der vom BMU herausgegebenen Zeitschrift Umwelt Nr. 6/1999 dokumentiert.

(5) Ministry of Environment and Energy/ Danish Environmental Protection Agency: Statement on „A product-oriented Environmental Initiative", Kopenhagen 1999 sowie Ministry of Environment and Energy/ Danish Environmental Protection Agency: Intensified Product-oriented Environmental Initiative, Kopenhagen 1999.

(6) DETR: Consumer Products and the Environment, London 1999.

(7) Dutch Ministry of Housing, Physical Planning and Environment: Product and the Environment. The implementation of the product-oriented policy. Letter from the Minister of Housing, Spatial Planning and the Environment, The Hague 1995.

(8) Government Communication: A Strategy for an Environmentally Sound Product Policy - Swedish Guidelines for an Integrated Product Policy, Stockholm 2000.

(9) Vgl. Rubik (Anm. 2).

\section{Der Autor}

Frieder Rubik ist wissenschaftlicher Mitarbeiter des IÖW im Regionalbüro Baden-Württemberg. Kontakt: IÖW, Bergstraße 7, 69120 Heidelberg, Tel. 06221/ 64916-0, Fax 06221/ 27060, E-mail: frieder.rubik@heidelberg.ioew.de 
(c) 20I0 Authors; licensee IÖW and oekom verlag. This is an article distributed under the terms of the Creative Commons Attribution Non-Commercial No Derivates License (http://creativecommons.org/licenses/by-nc-nd/3.o/), which permits unrestricted use, distribution, and reproduction in any medium, provided the original work is properly cited. 